\title{
Rapid Peptide Fragmentation without Electrons, Collisions, Infrared Radiation, or Native Chromophores
}

\author{
Geoffrey K. Yeh, Qingyu Sun, Claudia Meneses, and Ryan R. Julian \\ Department of Chemistry, University of California at Riverside, Riverside, California, USA
}

\begin{abstract}
Ultraviolet photodissociation of peptides followed by mass analysis has several desirable advantages relative to other methods, yet it has not found widespread use due to several limitations. One shortcoming is the inefficiency with which peptides absorb in the ultraviolet. This issue has a simple solution and can be circumvented by the attachment of noncovalent adducts that contain appropriate chromophores. Subsequent photoactivation of the chromophore leads to vibrational excitation of the complex and eventually to fragmentation of the peptide. Herein, the energetics that control the efficiency of this process are examined as a function of the characteristics of both the peptide and the noncovalently attached chromophore. Fragmentation efficiency decreases with increasing peptide size and is also constrained by the binding energy of the noncovalent adduct. The optimum chromophore should have excellent absorption at the excitation wavelength and a low luminescence quantum yield. It is demonstrated that a naphthyl based 18-crown-6 adduct is ideally suited for attaching to a variety peptides and fragmenting them following absorption of $266 \mathrm{~nm}$ light. Potential applications and limitations of this methodology are discussed. (J Am Soc Mass Spectrom 2009, 20, 385-393) (C 2009 Published by Elsevier Inc. on behalf of American Society for Mass Spectrometry
\end{abstract}

$\mathrm{T}$ There are a variety of mainstream fragmentation methodologies available for interrogating peptides, including collision-induced dissociation (CID) [1], infrared multiphoton photodissociation (IRMPD) [2], electron capture dissociation (ECD) [3], and electron-transfer dissociation (ETD) [4]. In addition, there are other methods that can be used, such as surface induced dissociation (SID) [5], black-body infrared radiative dissociation (BIRD) [6], along with various photodissociation experiments employing high-energy lasers [7-11]. Though none of these techniques is perfect, each has characteristic strengths and weaknesses. For example CID is easily employed in the source region of any instrument with atmospheric pressure ionization. CID is also easily accommodated elsewhere in an instrument as long as high vacuum is not required. In contrast, IRMPD is well suited for collisionless environments. In this regard, CID and IRMPD provide complementary capabilities. Furthermore, since both methods fragment ions by the stepwise addition of small amounts of energy, the results obtained are frequently quite similar.

However, CID and IRMPD are both limited by the fact that energy deposition occurs over a relatively long time scale, typically milliseconds to seconds. In con-

Address reprint requests to Dr. R. R. Juliian, Department of Chemistry, University of California, Riverside, Riverside, CA 92508, USA. E-mail: ryan.julian@ucr.edu trast, ultraviolet photodissociation (UVPD) occurs on a much shorter time scale and can yield fragment ions characteristic of CID or IRMPD. However, the major limitation of UVPD is that the precursor ion must contain a suitable chromophore. For peptides, UVPD requires either short wavelength lasers (less than 200 $\mathrm{nm}$ ) or peptides rich in tyrosine, tryptophan, and phenylalanine that can absorb at longer wavelengths. Therefore, UVPD is either limited to a small subset of all peptides or constrained by undesirable technical challenges which are encountered when working in the vacuum ultraviolet. Thus, widespread implementation of UVPD for peptide fragmentation has been to date somewhat limited.

However, Brodbelt and coworkers have recently described a method to circumvent the requirement for aromatic residues or short wavelength lasers [12]. In this work, a noncovalently attached chromophore was used to absorb energy from a photon and transfer it to a peptide, causing the peptide to fragment. 18-crown- 6 ether (18C6), which preferentially associates with lysine residues via the formation of three hydrogen bonds, was used for the noncovalent attachment [13-15]. When combined with a dipyrrolylquinoxaline chromophore, the crown/peptide interaction was found to accommodate vibrational energy transfer from the chromophore to the peptide. In this manner, energy can be transferred from the laser to the chromophore, and then from the chromophore to the peptide (following internal conver-
(C) 2009 Published by Elsevier Inc. on behalf of American Society for Mass Spectrometry. 1044-0305/09/\$32.00

doi:10.1016/j.jasms.2008.10.019
Published online November 5, 2008 Received August 26, 2008 Revised October 28, 2008 Accepted October 28, 2008 
sion into vibrational energy). Following excitation of the peptide, the crown adduct departs and the peptide fragments to yield products similar to those observed by CID.

Herein we report the discovery of a naphthyl based $18 \mathrm{C} 6$ reagent $(\mathrm{NC})$, which behaves similarly. Excitation of peptide/NC complexes results in efficient energytransfer to the peptide followed by fragmentation of the peptide. Importantly for NC adducts, a single laser pulse is found to be sufficient to induce fragmentation, meaning that CID like spectra can be obtained in a collisionless environment on a microsecond time scale. Further experiments were conducted to explore the issues that influence the degree of peptide fragmentation efficiency. The following factors were found to be important: the nature of the chromophore, the number of degrees of freedom in the peptide (or peptide size), and the energy gap between the barriers for noncovalent disassembly and covalent bond dissociation. Ideally, the chromophore should exhibit strong absorption and internal conversion. Fragmentation decreases as peptide size increases and as the amount of energy required for dissociating the peptide begins to significantly exceed the noncovalent binding energy. Nevertheless, under appropriate conditions peptides comprising a wide range of sizes and displaying substantial sequence diversity can be fragmented. This includes peptides that do not contain lysine and peptides exceeding $2000 \mathrm{Da}$. Furthermore, it is demonstrated that a single PD spectrum frequently contains fragments from multiple charge states, meaning that high information content is revealed in each spectrum.

\section{Materials and Methods}

$\mathrm{KP}_{13} \mathrm{~K}$ was synthesized by Genscript (Piscataway, NJ). Melittin, kassinin, Lys [0]bradykinin, bradykinin, dynorphin- $\beta$, GSK- $\beta$ inhibitor, myosin kinase inhibitor, and mouse ANP [1-11] were purchased from American Peptide Company (Sunnyvale, CA). Polyalanine peptides were synthesized in-house using standard Fmoc coupling methodology. Water was purified in-house to a minimum resistance of $18 \mathrm{M} \Omega$. HPLC grade acetonitrile $(\mathrm{ACN}), \mathrm{ACS}$ grade anhydrous tetrahydrofuran (THF), and ACS grade triethylamine (TEA) were purchased from Sigma Aldrich (St. Louis, MO).

NC and dansyl-crown (DC) were prepared by dissolving 2-hydroxymethyl-18-crown-6 (Sigma-Aldrich) in anhydrous THF containing a catalytic amount of triethylamine. 2-Napthoyl chloride or dansyl chloride (Sigma-Aldrich) were dissolved in THF and added dropwise to 2-hydroxymethyl-18-crown-6 solution. The reactions were cooled in an ice bath for 1 hour, and then left at room temperature for the following $18 \mathrm{~h}$. The products were purified by HPLC, filtered, evaporated under vacuum, and reconstituted in acetonitrile. The identities of the purified products were verified by mass spectrometry. Both nominal masses and fragmen- tation products from CID were consistent with the expected products.
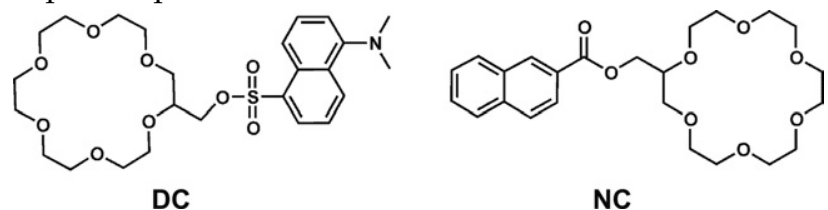

NC

Peptides were brought to $20 \mu \mathrm{M}$ in 60:40 acetonitrile: water. To these solutions, an equal volume of either pure acetonitrile, $40 \mu \mathrm{M}$ NC in acetonitrile, or $40 \mu \mathrm{M}$ DC in acetonitrile was added. This resulted in $10 \mu \mathrm{M}$ peptide in 80:20 acetonitrile:water for CID experiments and $10 \mu \mathrm{M}$ peptide and $20 \mu \mathrm{M}$ NC or DC in 80:20 acetonitrile:water for PD experiments. The ratio of peptide to crown does not tremendously influence the amount of adducts that are observed as noted previously [13], but the presence of sodium, potassium, or ammonia should be avoided by desalting. These solutions were infused by syringe pump at $2 \mu \mathrm{L} / \mathrm{min}$ into a Thermo Scientific LTQ linear ion trap mass spectrometer (Thermo, San Jose, CA). For both CID and UVPD experiments, the capillary temperature was set at $200{ }^{\circ} \mathrm{C}$.

\section{CID Experiments}

An isolation width of $3.5 \mathrm{Da}$ was chosen to isolate the precursor ion with satisfactory abundance and lack of interference in both CID and UVPD experiments. The isolation width was determined to have no significant influence on the degree of fragmentation by UVPD by comparing fragment ion spectra of complexes free of isobaric or near-isobaric interferences at varying isolation width settings. Activation parameters were set to default values: activation $Q(0.250)$, and activation time (30 ms).

The electrospray voltage ranged between 3.5 and 5.5 $\mathrm{kV}$ and was optimized to maximize ion transmission for each peptide. Other source and ion optics parameters were optimized using the auto tune function of the LTQ's Xcalibur software. Source conditions were optimized to allow maximum abundance of precursor ion for UVPD experiments. Of particular importance to the abundance of peptide/NC and peptide/DC complexes was the tube lens voltage. Decreasing the magnitude of this source parameter $(50-150 \mathrm{~V}$ in positive ion mode) often aided in maintaining the integrity of the noncovalent complexes for trapping, dissociation, and detection.

\section{UVPD Experiments}

The LTQ mass spectrometer used for these experiments was modified to accommodate the application of UV laser light into the linear ion trap as described previously [16]. Briefly, a quartz window was installed at the rear of the vacuum manifold, and a $266 \mathrm{~nm}$ NdYAG laser was aligned to direct light along the axis of the ion 
trap. A digital delay generator (Berkeley Nucleonics, San Rafael, CA) was used to precisely control the timing of the laser pulse with respect to a signal triggering the activation segment of the LTQ's MS ${ }^{n}$ scan cycle. In all UVPD experiments, a single $\sim 6 \mathrm{~mJ}$ pulse was used for photoactivation. The instrument is otherwise unmodified and the trap is expected to operate at typical pressures of around $\sim 1$ mTorr.

An isolation width of 3.5 Da was used for precursor ions. Activation parameters were set as follows: activation energy (0.0), activation $Q(0.100)$, and activation time (30 $\mu \mathrm{s}$, the minimum allowable time). The lowered activation $Q$ allows detection of low $m / z$ fragments useful in the analysis of small peptides, and the $30 \mu \mathrm{s}$ activation time exploits the exceptionally fast dissociation available by photoactivation of the isolated peptidenaphthyl-crown complex. No increase in product ion intensity was observed at $Q$ values higher than 0.1 , but reduced intensity was observed at settings below 0.08 . For both CID and UVPD experiments, 100 product ion scans were acquired and averaged.

The speed of the UVPD process was evaluated by isolating and photodissociating the $\left[\mathrm{KP}_{13} \mathrm{~K}+\mathrm{NC}+\right.$ $4 \mathrm{H}]^{4+}$ complex and scanning for the $\mathrm{b}_{4}$ ion over a $5 \mathrm{Da}$ scan range. The TurboScan $(66,000 \mathrm{Da} / \mathrm{s})$ scan rate was employed for this experiment so that the total scan time was $75 \mu \mathrm{s}$. The trigger from the digital delay generator was incrementally delayed to immediately precede ion ejection. The $b_{4}$ ion was observed to drop from $100 \%$ to $0 \%$ intensity in a span of under $1 \mu \mathrm{s}$. This probably corresponds with the amount of time needed to excite the ions out of the volume accessible to the laser, and may not reflect the ultimate speed of the process. In any case, the time required for photodissociation cannot be determined further within this limit in this instrument.

\section{Results and Discussion}

The electrospray mass spectrum for $\mathrm{NC}$ and $\mathrm{KP}_{13} \mathrm{~K}$ is shown in Figure 1a. The voltages in the source region of the instrument must be tuned to allow for the transmission of noncovalent complexes as detailed in the experimental section above. Once this is achieved, peptide/NC complexes are typically detected with high abundance as demonstrated in Figure 1a. Isolation of the $[\mathrm{M}+\mathrm{NC}+4 \mathrm{H}]^{4+}$ peak, followed by photoactivation yields the spectrum shown in Figure 1b. Simple dissociation of the noncovalent bond to yield the $[\mathrm{M}+$ $3 \mathrm{H}]^{3+}$ peak is observed ( $\mathrm{NC}$ departs with a charge) in addition to many fragments that correspond to $b$ and $y$ ions. The spectrum shown in Figure $1 b$ is not magnified in any way and was obtained with a single laser pulse (all spectra shown herein are from a single laser pulse), indicating that adduct assisted PD is very efficient for this peptide. Photoactivation of the peptide in the absence of NC is shown in Figure 1c. In this case, no significant peptide dissociation is observed, revealing that complexation with $\mathrm{NC}$ is entirely responsible for the fragmentation observed in Figure $1 \mathrm{~b}$. These results
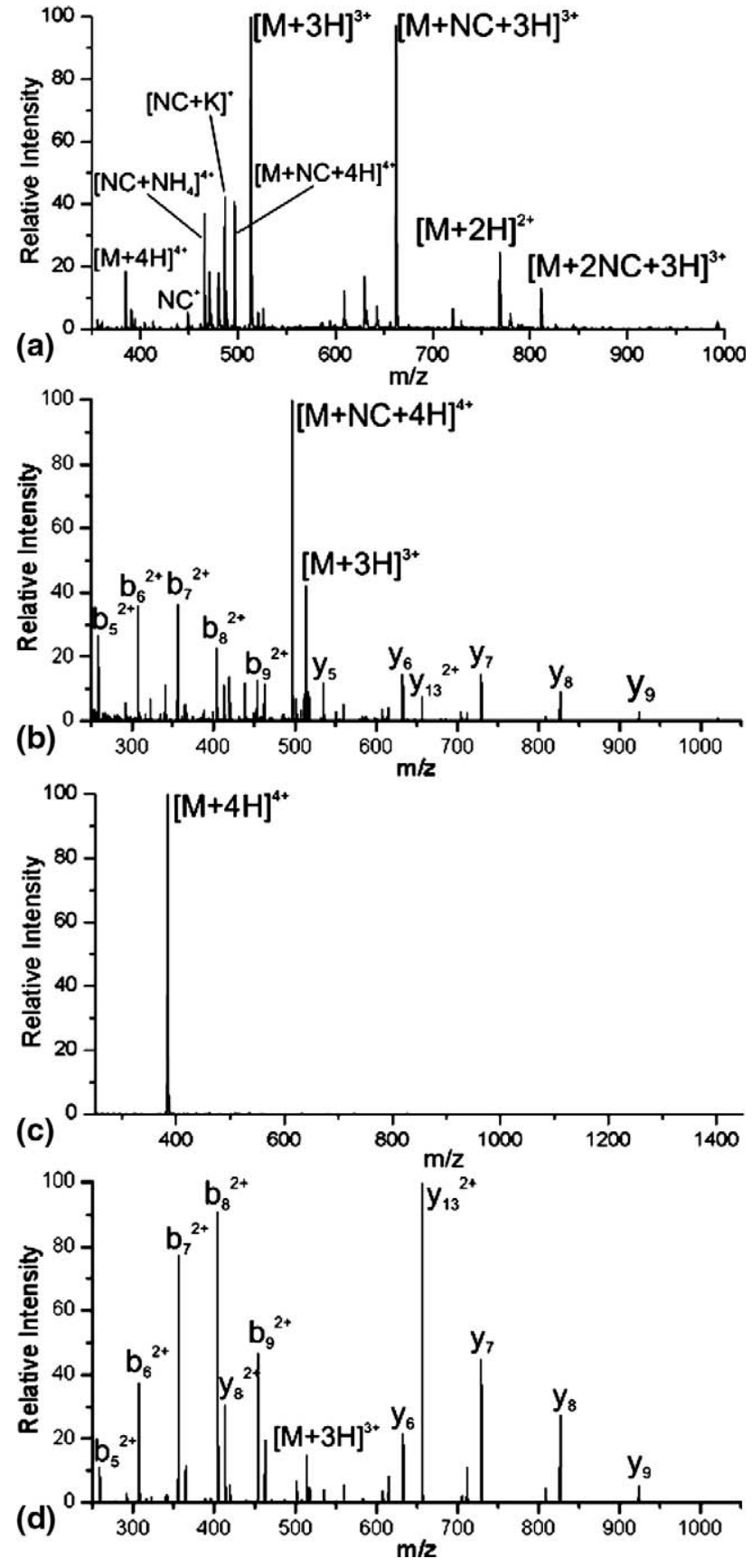

Figure 1. (a) ESI mass spectrum for $\mathrm{NC}$ and $\mathrm{KP}_{13} \mathrm{~K}$, demonstrating abundant adduct formation. (b) PD spectrum for $[\mathrm{M}+\mathrm{NC}+$ $4 \mathrm{H}]^{4+}$. Abundant CID-like fragments are observed. (c) Photoactivation of the peptide itself yields no fragments. (d) CID spectrum for $[\mathrm{M}+3 \mathrm{H}]^{3+}$.

agree with previous experiments demonstrating that peptides must contain aromatic residues to absorb well at $266 \mathrm{~nm}$ [10]. The typical CID spectrum for $\mathrm{KP}_{13} \mathrm{~K}$ is shown in Figure 1d. This spectrum is very similar to that shown in Figure 1b, suggesting that a statistically driven process yields both products. Since it is well known that CID in an ion trap occurs following vibrational excitation [17], it implies the fragments shown in Figure $1 \mathrm{~b}$ were also created after vibrational heating. 
For the peptide in Figure $1 \mathrm{~b}$ to be "heated" by a 266 $\mathrm{nm}$ laser, a fairly complex process must take place. First, the naphthyl group must absorb a photon, generating an excited electronic state. This energy can be released by luminescence or converted into vibrational excitation. Luminescence will lead to no observable effect on the mass spectrum because the photon energy will simply be lost to the surrounding environment [18]. In contrast, relaxation of the excited-state to the ground state by vibronic coupling will lead to vibrationally excited NC. Thus, chromophores which favor internal conversion should be selected for this type of experiment. This selection is not a trivial task since the gas-phase behavior of chromophores does not necessarily follow that observed in solution [19, 20], and gasphase properties have not been nearly as well characterized. Following internal conversion of the photon energy, internal vibrational energy redistribution (IVR) [21], which redistributes energy among a network of covalently bound atoms, typically takes place faster than vibrational energy-transfer (VET), which occurs through noncovalent bonds [22]. IVR typically occurs on the picosecond time scale, with VET occurring approximately an order of magnitude slower [22]. Therefore, the NC should become vibrationally excited before energy begins to transfer to the peptide. Nevertheless, the vibrationally hot NC should easily transfer energy to the peptide within the nanosecond time scale, leading to a vibrationally excited complex. At this point, the system can be thought of as a special case of unimolecular dissociation where the complex is treated as a molecule. The timescale for fragmentation will be dictated by the amount of energy in the peptide and the number of modes into which that energy can be partitioned. We have measured dissociation occurring in as little as $1 \mu \mathrm{s}$ in our ion trap by delaying the laser pulse to immediately precede ion detection. Furthermore, statistical fragmentation dictates that the weakest bonds should be broken to yield the most abundant products [23]. In these experiments, it is assumed that the weakest bonds are the noncovalent bonds holding the complex together. Indeed, fracture of the noncovalent interaction yields the most abundant fragment in most cases. In order for the peptide itself to fragment, sufficient energy must remain in the peptide after loss of NC. Alternatively, the peptide may fragment while still complexed to the NC, followed by subsequent loss of NC. The detection of trace amounts of peptide fragments with NC still attached suggests that both processes likely occur (see supplemental information, which can be found in the electronic version of this article). The energetics relevant to this process are explored further below.

\section{Other Chromophores}

To compare the effectiveness of several chromophores, we synthesized a dansyl crown (DC) and examined a peptide which was previously evaluated by Brodbelt and coworkers (KRPPGFSPFR) [12]. In addition, we evaluated the fragmentation efficiency in a peptide with a native covalently bound chromophore, tryptophan. The results are shown in Figure 2. The dissociation observed with DC and NC is quite comparable (Figure $2 a$ and $b$, respectively). Fragmentation is also similar to that obtained previously for the same peptide at $355 \mathrm{~nm}$ with the dipyrrolylquinoxaline chromophore [12].
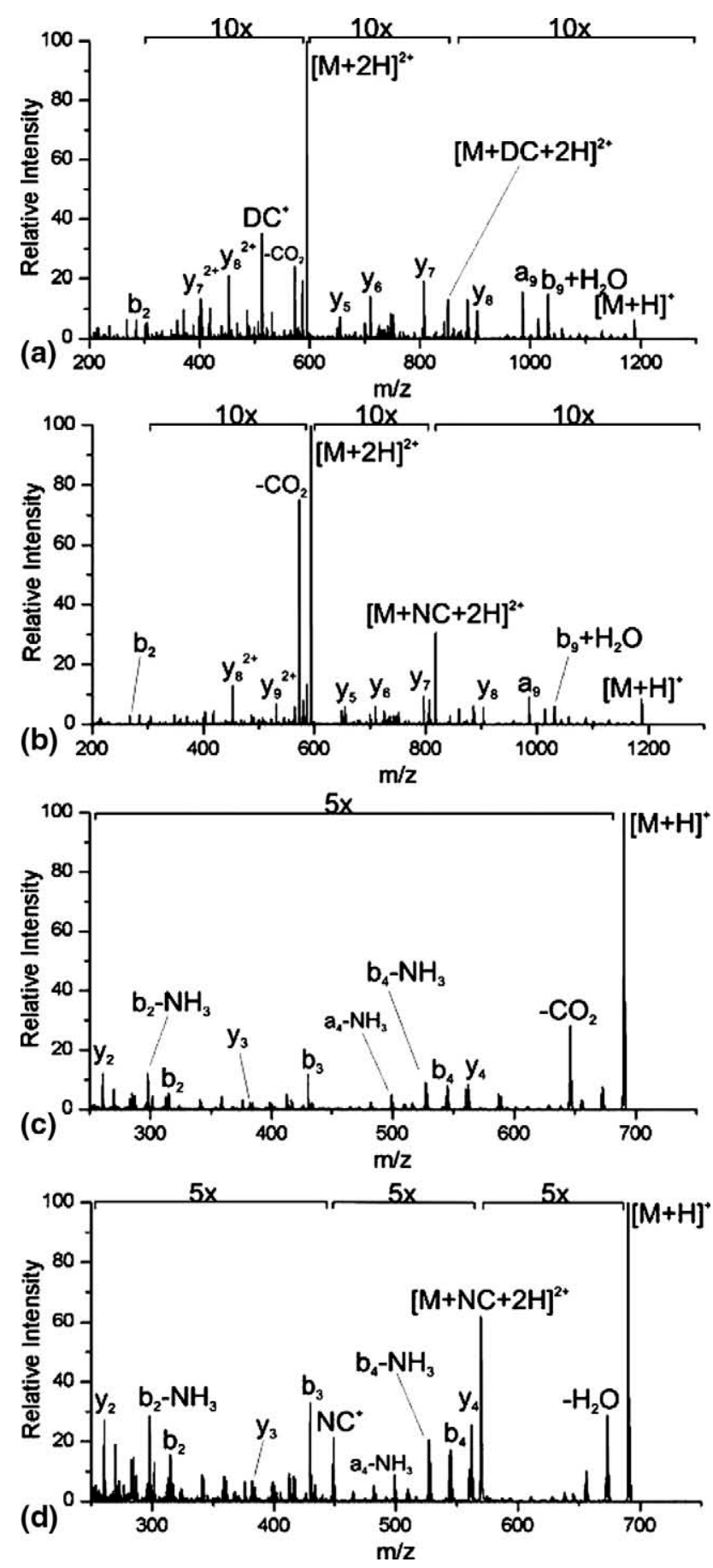

Figure 2. PD spectra for KRPPGFSPFR with (a) DC and (b) NC yield very similar results. The fragmentation efficiency for a tryptophan containing peptide, KWDNQ, without (c) and with NC (d) are compared. 
However, fragmentation is clearly superior at $266 \mathrm{~nm}$ for DC and NC compared to dipyrrolylquinoxaline. This observation most likely relates to relative absorption maxima for the respective dyes, which is favored at shorter wavelengths for NC and DC. These results suggest that a variety of dyes should be suitable for these experiments and that the specific choice should be dictated by laser wavelengths that are available for excitation. In Figure $2 \mathrm{c}$ and $\mathrm{d}$, the fragmentation efficiency is compared for a tryptophan containing peptide by itself and with NC attached. The fragmentation efficiency is notably better with the addition of NC. There are several potential explanations for this observation. Possibly the absorption efficiency is better or the fluorescence intensity is reduced for the NC relative to tryptophan. Alternatively, there may be a multiplicative effect on fragmentation from the presence of two chromophores, although this situation is not observed to aid fragmentation of peptides with multiple noncovalently attached chromophores (see supplemental information).

\section{Size Matters}

Experiments with a series of polyalanine peptides are shown in Figure 3. Polyalanine was chosen as the model to examine the effect of peptide size to minimize the effects that sequence or charge state diversity might introduce into the fragmentation process. Photodissociation of three representative polyalanines is shown in Figure $3 a-c$. Inspection of the raw data reveals that the abundance of unfragmented $[\mathrm{M}+\mathrm{H}]^{+}$relative to peptide fragments grows larger with increasing peptide size. This suggests that less dissociation occurs as peptide size increases. The data for the entire polyalanine series is quantified in Figure $3 \mathrm{~d}$, where the amount of fragmentation is shown as function of the number of degrees of freedom in the peptide. Fragmentation efficiency is quantified by the ratio of the sum intensity of all peptide fragments to the intensity of the $[\mathrm{M}+\mathrm{H}]^{+}$ ion. Normalizing the data in this way eliminates variations in fragment intensity which may result from changes in the total laser power or absorption efficiency. Figure $3 \mathrm{~d}$ shows a clear trend favoring less dissociation as the number of degrees of freedom increases, which can be simply explained. The amount of energy per photon is not changing, which means that the amount of energy per degree of freedom decreases as peptide size increases. Statistically, this will lead to a decrease in the rate of fragmentation and therefore fewer fragments will be observed. Size is therefore an important factor that will influence the degree of fragmentation that is observed in this type of experiment. Nevertheless, fairly large peptides can be fragmented under favorable circumstances as described further below.
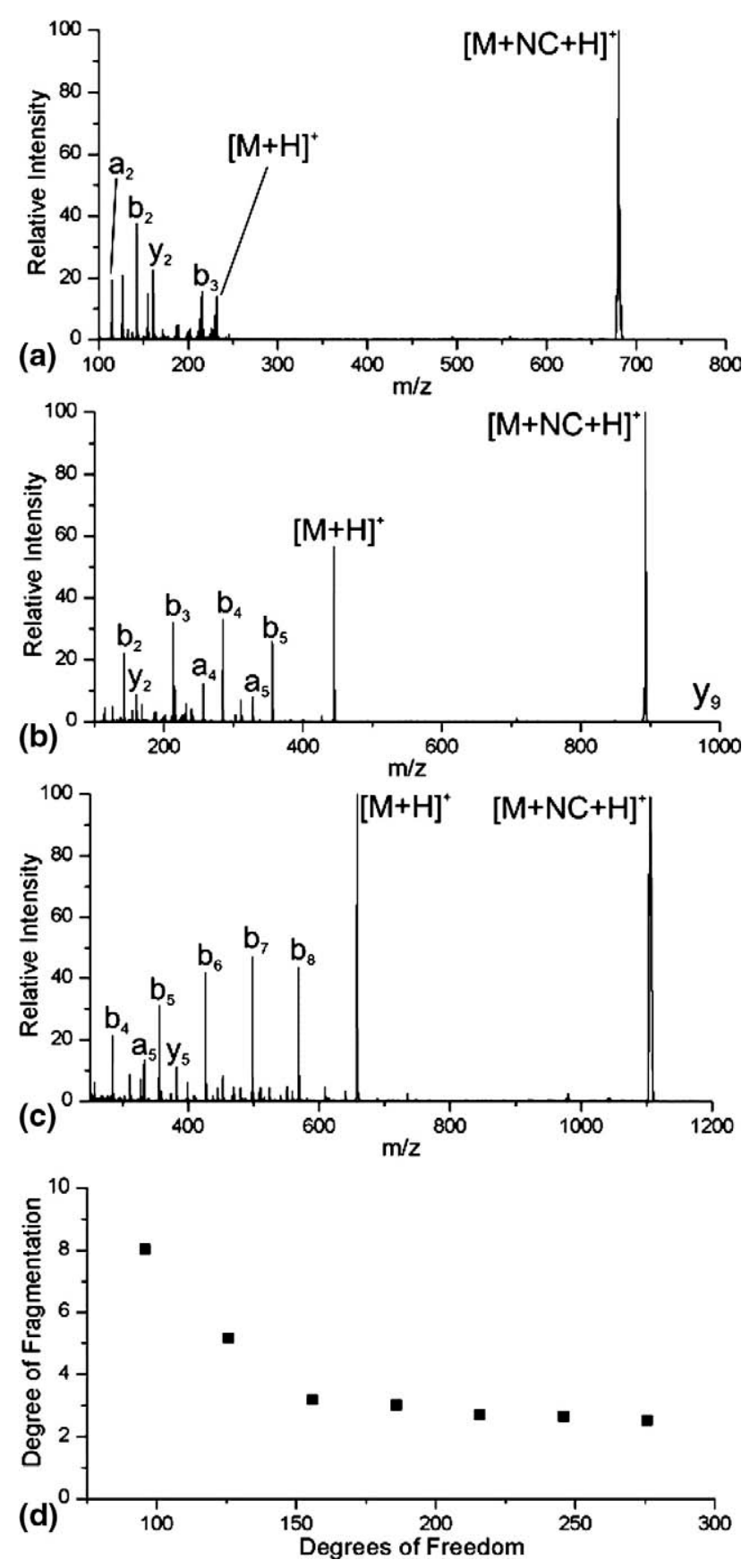

Figure 3. PD spectra for (a) $\left[\mathrm{A}_{3}+\mathrm{NC}+\mathrm{H}\right]^{+}$, (b) $\left[\mathrm{A}_{6}+\mathrm{NC}+\right.$ $\mathrm{H}]^{+}$, and $(\mathrm{c})\left[\mathrm{A}_{9}+\mathrm{NC}+\mathrm{H}\right]^{+}$. Fragmentation decreases with increasing peptide size. (d) The degree of fragmentation is quantified for the entire series of peptides $\mathrm{A}_{3}-\mathrm{A}_{9}$. The ratio is defined as the sum intensity of all peptide fragments to the intensity of [M+ $\mathrm{H}]^{+}$. The intensity of the NC/peptide complex is not included.

\section{Relevant Energetics}

The use of noncovalent adducts which act as the chromophores in these experiments introduces energetic constraints that would not be present in a covalently bound system. To explain, the amount of energy contained in a $266 \mathrm{~nm}$ photon $(\sim 4.6 \mathrm{eV})$ is relatively small compared to the amount of energy required to fragment 
even a modestly sized peptide. Previous calculations using RRKM theory have estimated that $15 \mathrm{eV}$ are required to fragment $\mathrm{Gly}_{10}$ at a reasonable rate [23]. More recent work suggests that even more energy may be required, particularly at short timescales [24]. Therefore, fragmentation in the present experiments is only feasible for a subpopulation of ions which are already vibrationally excited before absorption of a photon. For example, Gly I $_{10}$ with $14 \mathrm{eV}$ of vibrational energy would meet these requirements. Following photoactivation and partitioning of the additional energy between NC and $\mathrm{Gly}_{10}, \sim 2.5 \mathrm{eV}$ would be added to $\mathrm{Gly}_{10}$ for a total of $\sim 16.5 \mathrm{eV}$. In this example, the NC departs with a significant fraction of the energy because Gly ${ }_{10}$ and NC are comparable in size; however, at larger peptide sizes more of the energy on average will remain with the peptide. Furthermore, regardless of the peptide size, the initial temperature of the complex must also be sufficiently cool to avoid disassembly of the noncovalent bonds holding the complex together. Therefore, the active subpopulation must be thermally excited, but not to the point where noncovalent bonds are broken at a significant rate. Since the photon delivers only a set amount of energy, this suggests that only fragmentation processes that are near the noncovalent dissociation threshold (which is $\sim 1.5 \mathrm{eV}$ for $18 \mathrm{C} 6$ based interactions) [13], will be easily accessible. Otherwise, insufficient energy will be added to significantly enhance the rate of dissociation. This concept is shown schematically in Figure S1 in the supporting information and can be explored by comparing the CID spectra for the peptide/NC complexes with the matching PD spectra.

PD and CID spectra for NC complexed with kassinin $\left(\right.$ DVPKSDQFVGLM-NH ${ }_{2}$ ) are shown in Figure $4 a$ and $\mathrm{b}$, respectively. The base peak in Figure $4 \mathrm{a}$ corresponds to the originally isolated complex, indicating that a large portion of the complex does not fragment in any way (presumably due to insufficient total vibrational energy). When fragmentation does occur, the primary product is intact peptide; however, fragments corresponding to $\mathrm{b}$ and $\mathrm{y}$ ions accompanied by several internal fragments are produced in high yield relative to the intact peptide. The fragmentation efficiency as defined previously is given in Table 1 . The fragments in Figure 4a match those produced by CID of the kassinin itself (see supplemental information). CID of the peptide complex (as shown in Figure 4 b) yields complex disassembly and concurrent fragmentation of kassinin. This suggests that the amount of energy required to fragment the complex and the amount of energy required to fragment the peptide itself are comparable in magnitude because the addition of energy by CID occurs over a long time scale in very small increments in an ion trap. Thus, the additional vibrational energy provided by a single photon is sufficient to cause disassembly of the complex and fragmentation of the peptide.

An example that contrasts this behavior is found with bradykinin and NC. Bradykinin (RPPGFSPFR) is
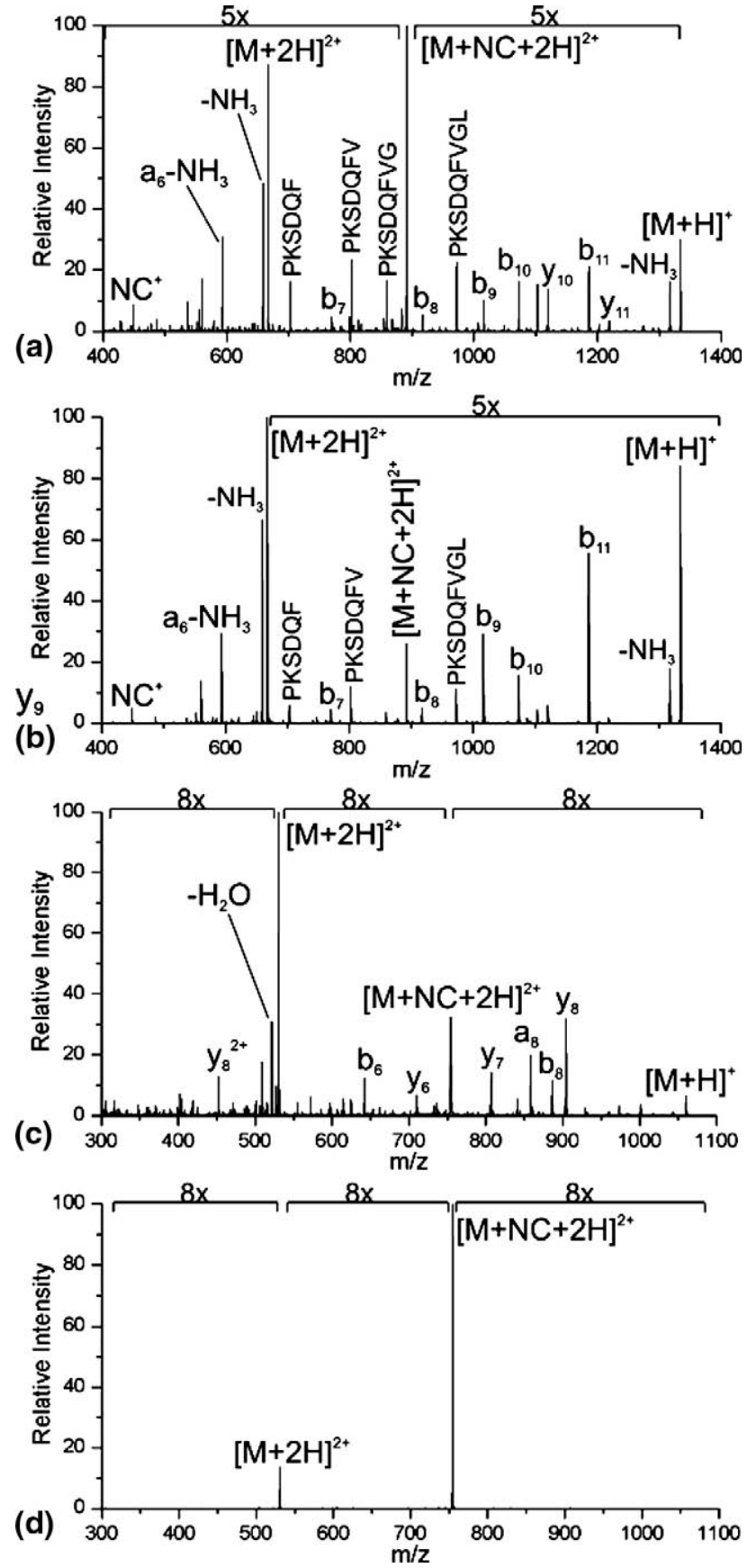

Figure 4. (a) PD spectrum for kassinin with NC. (b) CID spectrum for kassinin with NC. (c) PD spectrum for bradykinin with NC. (d) CID spectrum for bradykinin with NC. The degree of fragmentation is correlated with the energy gap between noncovalent disassembly and backbone fracture.

an arginine containing peptide, which is frequently encountered in gas-phase experiments. The photodissociation spectrum for $[\mathrm{M}+\mathrm{NC}+2 \mathrm{H}]^{2+}$ is shown in Figure 4c. Dissociation of the complex is very efficient, but yield of peptide fragments is smaller than was observed with kassinin. The fragments that are observed are very similar to those generated by CID (see supplemental information). In addition, similar results are obtained with the $[\mathrm{M}+\mathrm{NC}+3 \mathrm{H}]^{3+}$ complex, 
Table 1. Degree of fragmentation ${ }^{\mathrm{a}}$ by PD

\begin{tabular}{lccc}
\hline Peptide & $\begin{array}{c}\text { Fragmentation } \\
\text { efficiency }\end{array}$ & $\begin{array}{c}\text { Degrees of } \\
\text { freedom }\end{array}$ & $\begin{array}{c}\text { Charge } \\
\text { state }\end{array}$ \\
\hline \hline $\mathrm{KP}_{13} \mathrm{~K}$ & 7.9 & 684 & $4+$ \\
Kassinin & 1.6 & 567 & $2+$ \\
Bradykinin & 0.3 & 450 & $2+$ \\
Melittin & 0.8 & 1293 & $3+$ \\
Melittin & 5.7 & 1299 & $5+$ \\
\hline
\end{tabular}

aDefined as the ratio of the sum intensity of all peptide fragments to the intensity of the precursor peptide.

although the degree of peptide fragmentation does not improve with an additional charge. CID of the bradykinin complex with NC yields only dissociation of noncovalent bonds as shown in Figure $4 \mathrm{~d}$. It is not possible to add sufficient energy into the complex by collisional excitation to fragment the peptide before loss of NC. This suggests that there is a significant gap between the amount of energy required to fragment the complex and the amount of energy required to fragment the peptide itself. This gap allows all of the NC to be lost before the peptide is sufficiently heated to fragment itself when subjected to CID. Similarly, the end result is poor fragmentation of bradykinin by PD because the additional energy from the photon is only sufficient to fragment the hottest subpopulation of peptides.

Unfortunately, there are several other important differences between bradykinin and kassinin. They are different sizes and contain different amino acids. Potentially important is the fact that kassinin contains lysine (the preferred binding site for 18C6) [13] and bradykinin does not, which could potentially influence the efficiency of VET. To eliminate the effects that such differences might cause, further experiments were carried out with melittin. The results for PD and CID experiments with melittin are shown in Figure 5. In particular, the +3 and +5 charge states are examined. The additional protons facilitate a lower energy fragmentation pathway for the +5 charge state, allowing comparison of the degree of fragmentation for two peptides where the sequence and size are effectively the same. As shown in Figure 5a, the +3 charge state does not fragment to a significant extent following photoactivation. Similarly, the spectrum shown in Figure $5 b$ reveals that simple disassembly of the noncovalent complex occurs by CID. These results are consistent with a significant energy gap between the noncovalent binding energy and the barriers to fragmentation of the peptide. In contrast, significantly more PD of the peptide is observed in Figure $5 c$ for the +5 charge state (a quantitative comparison is shown in Table 1). At the same time, CID yields fragmentation of both noncovalent and covalent bonds as shown in Figure 5d. The binding energy of NC to melittin should not be substantially modified by the addition of two protons; however, the addition of protons frequently enables lower energy dissociation pathways. The results in
Figure 5 clearly show that narrowing the gap between these two quantities results in increased peptide fragmentation, even when all other factors are held constant. The results in Figure $5 c$ also demonstrate that peptides of significant size can be fragmented by UVPD.
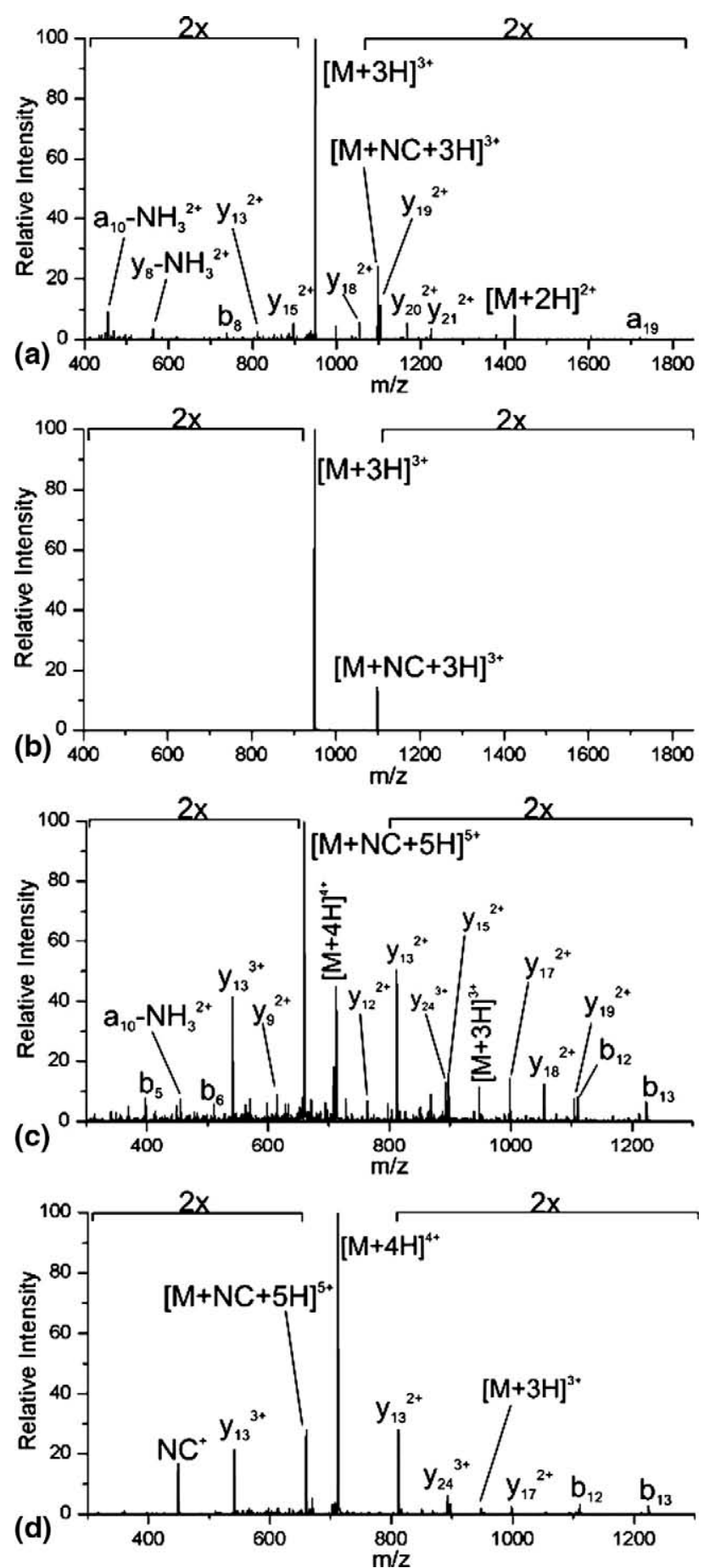

Figure 5. (a) PD spectrum for melittin $3+$ with NC. (b) CID spectrum for melittin 3+ with NC. (c) PD spectrum for melittin 5+ with NC. (d) CID spectrum for melittin 5+ with NC. The degree of fragmentation is again correlated with the energy gap between noncovalent disassembly and backbone fracture. In this case, other potentially influencing factors are held constant. 


\section{Nonthermal Fragmentation}

Most peptides that we have examined by PD exhibit fragmentation behavior very similar to CID; however, in a few cases, fragments that are not typically observed by CID were found. For example in Figure 6a, PD of
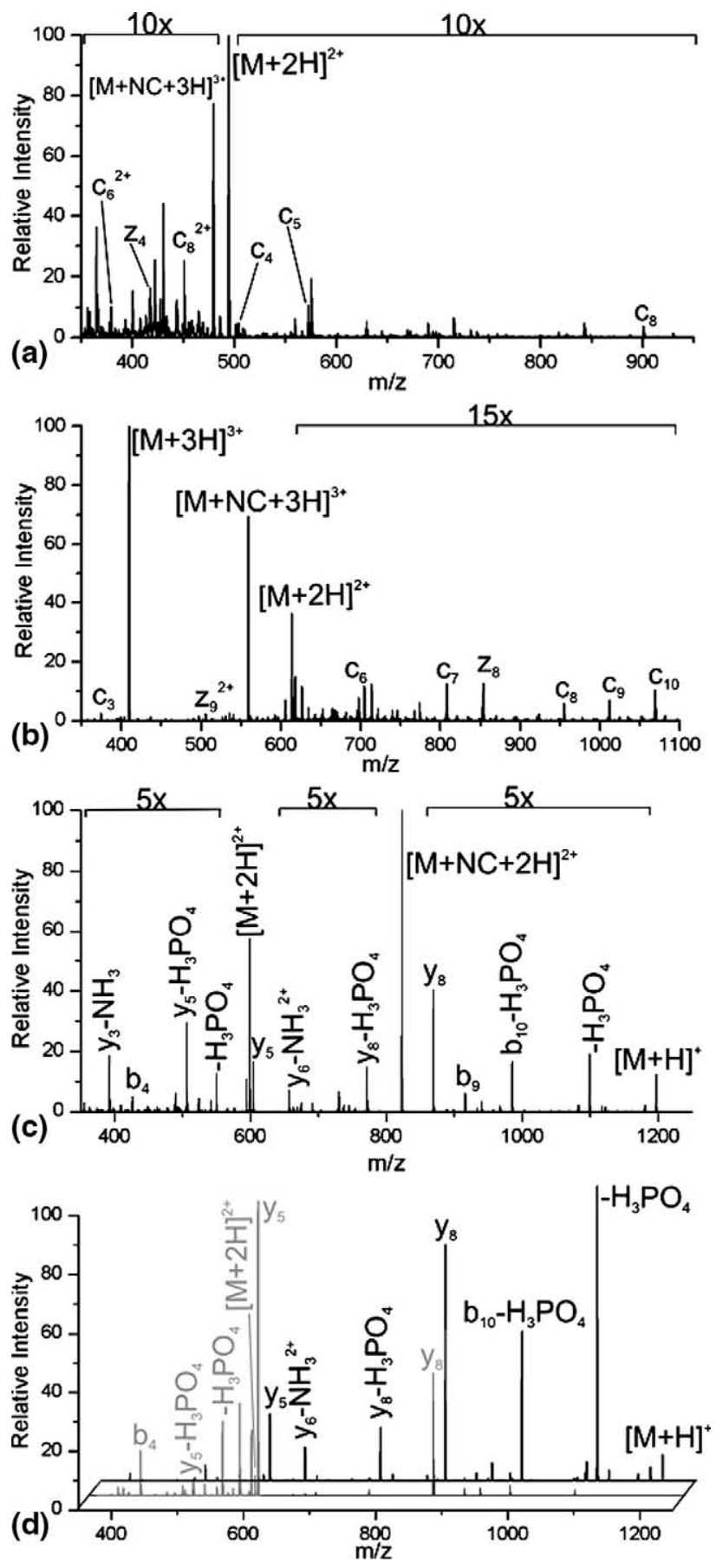

Figure 6. (a) PD spectrum for [KKRAARATS-NH $\mathrm{H}_{3}+\mathrm{NC}+$ $3 \mathrm{H}]^{3+}$ yields unusual fragments. (b) Similar results are obtained by PD of [SLRRSSCFGGR $+\mathrm{NC}+3 \mathrm{H}]^{3+}$. (c) PD spectrum for [KEAPPAPPQsP $\left.-\mathrm{NH}_{3}+\mathrm{NC}+3 \mathrm{H}\right]^{3+}(\mathrm{s}=$ phosphorylated serine). (d) CID spectra for the +1 and +2 charge states of KEAPPAPPQsP $-\mathrm{NH}_{3}$. Fragments from both charge states are found in (c). $\left[\text { KKRAARATS- } \mathrm{NH}_{3}+\mathrm{NC}+3 \mathrm{H}\right]^{3+}$ yields primarily c and $\mathrm{z}$ ions, which are reminiscent of electron capture experiments. The CID spectrum for this peptide does not yield similar fragments (see supplemental information). The peptide SLRRSSCFGGR behaves similarly, as shown in Figure $6 \mathrm{~b}$. A series of $\mathrm{c}$ and $\mathrm{z}$ ions is again produced by PD, which are not observed in the CID spectra for the peptide or for the peptide/NC complex (see supplemental information). What is the origin of the $c$ and $z$ ions in Figure $6 a$ and $b$ ? We do not have a definitive answer for this question. Possibly, the laser could generate free electrons upon contacting metal electrodes within the ion trap; however, given the strong electrical fields that are present in all parts of the instrument, it is unlikely that electron capture could efficiently take place. Possibly, the excited-state naphthyl group could react via electron-transfer with these peptides to generate $c$ and $\mathrm{z}$ ions. If this were the case, there must be structural features that facilitate electron transfer for these peptides relative to most peptides. Further experiments will be needed to evaluate these possibilities.

\section{PD Instead of CID}

For simplicity sake, CID will be the more pragmatic choice for fragmenting peptides in most experiments. However, there are situations where CID may not be the optimum choice. For example, experiments where fragmentation is desired very rapidly are not amenable to CID. High-energy collisions (which are required to reduce the timescale) are more difficult to arrange and lead to greater ion scattering and reduced sensitivity. In contrast, UVPD naturally occurs very quickly on the time scale of mass spectrometry. In this regard, UVPD may be useful in TOF-TOF experiments where rapid fragmentation is desirable. An additional benefit for this experiment is that no collision gas is required. Another advantage of this particular form of UVPD stems from the disassembly of the noncovalent complex which is demonstrated in Figure $6 c$ and d. To explain, multiply charged peptide complexes are typically examined. When the NC departs, this frequently occurs by loss of both protonated and neutral NC, generating multiple charge states of the precursor peptide. Therefore, the PD spectrum in Figure $6 \mathrm{c}$ contains fragments from both the singly and doubly charged precursor peptide. This is demonstrated in Figure 6d, where overlapping of both CID spectra are required to reproduce the peaks in Figure 6c. In addition, there are a few peaks (such as $\mathrm{y}_{3}-\mathrm{NH}_{3}$ in Figure 6c) that are typical CID peaks, yet are not observed in the CID spectra. These peaks may originate from unique dissociation pathways that are only accessible with NC still attached. The end result is that a single UVPD spectrum can yield information that would typically be obtained from multiple CID experiments, and UVPD does not require a priori knowledge of the locations of the various charge states to accomplish this task. 


\section{Conclusions}

Clearly, noncovalently attached chromophores can be used to fragment peptides by UVPD, circumventing the problem of low inherent peptide absorption. PD can be observed after a single laser pulse on the microsecond timescale, typically yielding fragments similar to those obtained by CID. Peptide size and the amount of energy required to fragment the peptide backbone are important determinants for the degree of fragmentation that is observed. Overall, the method is constrained by incomplete fragmentation of the precursor peptide; however, for applications requiring rapid dissociation in a collisionless environment, noncovalently assisted UVPD may find use. Furthermore, additional optimization of the chromophore and recognition motif for the noncovalent adducts may lead to more abundant peptide fragmentation in future experiments.

\section{Acknowledgments}

The authors thank the University of California at Riverside and the NSF (CHE-0747481) for financial support. They also thank Peter Armentrout and Daniel Austin for valuable discussions.

\section{References}

1. McLuckey, S. A. Principles of Collisional Activation in Analytical Mass-Spectrometry. I. Am. Soc. Mass Spectrom. 1992, 3(6), 599-614.

2. Little, D. P.; Speir, J. P.; Senko, M. W.; O'Connor, P. B.; McLafferty, F. W. Infrared Multiphoton Dissociation of Large Multiply-Charged Ions for Biomolecule Sequencing. Anal. Chem. 1994, 66(18), 2809-2815.

3. Zubarev, R. A.; Horn, D. M.; Fridriksson, E. K.; Kelleher, N. L.; Kruger, N. A.; Lewis, M. A.; Carpenter, B. K.; McLafferty, F. W. Electron Capture Dissociation for Structural Characterization of Multiply Charged Protein Cations. Anal. Chem. 2000, 72(3), 563-573.

4. Syka, J. E. P.; Coon, J. J.; Schroeder, M. J.; Shabanowitz, J.; Hunt, D. F. Peptide and Protein Sequence Analysis by Electron Transfer Dissociation Mass Spectrometry. Proc. Natl. Acad. Sci. U.S.A. 2004, 101(26), 9528-9533.

5. Wysocki, V. H.; Joyce, K. E.; Jones, C. M.; Beardsley, R. L. SurfaceInduced Dissociation of Small Molecules, Peptides, and Noncovalent Protein Complexes. J. Am. Soc. Mass Spectrom. 2008, 19(2), 190-208.

6. McLuckey, S. A.; Goeringer, D. E. Slow Heating Methods in Tandem Mass Spectrometry. J. Mass Spectrom. 1997, 32(5), 461-474.
7. Thompson, M. S.; Cui, W. D.; Reilly, J. P. Fragmentation of Singly Charged Peptide Ions by Photodissociation at $\lambda=157 \mathrm{~nm}$. Angew. Chem. Int. Ed. 2004, 43(36), 4791-4794.

8. Thompson, M. S.; Cui, W. D.; Reilly, J. P. Factors That Impact the Vacuum Ultraviolet Photofragmentation of Peptide Ions. J. Am. Soc. Mass Spectrom. 2007, 18(8), 1439-1452.

9. Lemoine, J.; Tabarin, T.; Antoine, R.; Broyer, M.; Dugourd, P. UV Photodissociation of Phospho-Seryl-Containing Peptides: Laser Stabilization of the Phospho-Seryl Bond with Multistage Mass Spectrometry. Rapid Commun. Mass Spectrom. 2006, 20(3), 507-511.

10. Oh, J. Y.; Moon, J. H.; Kim, M. S. Chromophore Effect in Photodissociation at $266 \mathrm{~nm}$ of Protonated Peptides Generated by Matrix-Assisted Laser Desorption Ionization (MALDI). J. Mass Spectrom. 2005, 40(7), 899-907.

11. Moon, J. H.; Yoon, S. H.; Kim, M. S. Photodissociation of Singly Protonated Peptides at $193 \mathrm{~nm}$ Investigated with Tandem Time-ofFlight Mass Spectrometry. Rapid Commun. Mass Spectrom. 2005, 19(22), 3248-3252.

12. Wilson, J. J.; Kirkovits, G. J.; Sessler, J. L.; Brodbelt, J. S. Photodissociation of Noncovalent Peptide-Crown Complexes. I. Am. Soc. Mass Spectrom. 2008, 19, 257-260.

13. Julian, R. R.; Beauchamp, J. L. Site Specific Sequestering and Stabilization of Charge in Peptides by Supramolecular Adduct Formation with 18-Crown-6 Ether by Way of Electrospray Ionization. Int. J. Mass Spectrom. 2001, 210(1/3), 613-623.

14. Julian, R. R.; May, J. A.; Stoltz, B. M.; Beauchamp, J. L. Molecular Mousetraps: Gas-Phase Studies of the Covalent Coupling of Noncovalent Complexes Initiated by Reactive Carbenes Formed by Controlled Activation of Diazo Precursors. Angew. Chem. Int. Ed. 2003, 42(9), 1012-1015.

15. Ly, T.; Julian, R. R. Using ESI-MS to Probe Protein Structure by Site-Specific Noncovalent Attachment of 18-Crown-6. J. Am. Soc. Mass Spectrom. 2006, 17(9), 1209-1215.

16. Ly, T.; Julian, R. R. Residue-Specific Radical-Directed Dissociation of Whole Proteins in the Gas Phase. J. Am. Chem. Soc. 2008, 130(1), 351-358.

17. Laskin, J.; Futrell, J. H. Activation of Large Ions in FT-ICR Mass Spectrometry. Mass Spectrom. Rev. 2005, 24(2), 135-167.

18. Dunbar, R. C. Photodissociation of Trapped Ions. Int. J. Mass Spectrom. 2000, 200(1/3), 571-589.

19. Khoury, J. T.; Rodriguez-Cruz, S. E.; Parks, J. H. Pulsed Fluorescence Measurements of Trapped Molecular Ions with Zero Background Detection. J. Am. Soc. Mass Spectrom. 2002, 13(6), 696-708.

20. Iavarone, A. T.; Duft, D.; Parks, J. H. Shedding Light on Biomolecule Conformational Dynamics Using Fluorescence Measurements of Trapped Ions. J. Phys. Chem. A 2006, 110(47), 12714-12727.

21. Stannard, P. R.; Gelbart, W. M. Intramolecular Vibrational-Energy Redistribution. J. Phys. Chem. 1981, 85(24), 3592-3599.

22. Assmann, J.; Kling, M.; Abel, B. Watching Photoinduced Chemistry and Molecular Energy Flow in Solution in Real Time. Angew. Chem. Int. Ed. 2003, 42(20), 2226-2246.

23. Marzluff, E. M.; Beauchamp, J. L. Collisional Activation Studies of Large Molecules. In Large Ions: Their Vaporization, Detection, and Structural Analysis, Baer T, Ed.; John Wiley and Sons: New York, 1996; p. 115-143.

24. Laskin, J.; Yang, Z.; Chu, I. K. Energetics and Dynamics of Electron Transfer and Proton Transfer in Dissociation of MetalII(Dalen) -Peptide Complexes in the Gas Phase. J. Am. Chem. Soc. 2008, 130(10), 3218-3230. 